\title{
3D Fluid-Structure Interaction simulation of a bileaflet heart valve opening-closing cycle and comparison with experimental flow data
}

\author{
D. Palmieri ${ }^{1}$, G. Vezzani ${ }^{1}$, F. Lagasco ${ }^{1} \& \mathrm{~S}$. Pascale $^{2}$ \\ ${ }^{I} D^{\prime}$ Appolonia S.p.A., Italy \\ ${ }^{2}$ Sorin Biomedica Cardio s.r.l., Italy
}

\begin{abstract}
A fully automated numerical procedure has been implemented to enable 3D modelling of the physical behaviour of blood flow through an implanted Mechanical Heart Valve (MHV). For this purpose, a specifically developed Valve Cycle Program was coupled with a commercial Computational Fluid Dynamics (CFD) solver to implement a Fluid-Structure Interaction (FSI) model. A complete opening-closing cycle has been simulated by imposing physiological boundary conditions. A parallel laboratory circulatory mock-loop hosting a MHV has been manufactured to carry out experimental Particle Image Velocimetry (PIV) flow measurements. The predicted flow characteristics were found to be in good agreement with experimental observations. Both numerical and experimental results confirmed that flow patterns and associated shear stresses downstream of the valve result in a low thrombosis and haemolysis potential.
\end{abstract}

Keywords: heart valve, CFD, Fluid-Structure Interaction, PIV.

\section{Introduction}

Since the first successful implantation of a prosthetic heart valve four decades ago, over 50 different designs have been developed including both mechanical and bioprosthetic valves. Today, with over 200,000 implants worldwide each year, the most widely implanted design is the mechanical bileaflet prosthesis [1] However, MHV implants suffer from complications resulting from thrombus deposition and patients implanted with these valves need to withstand long-term anti-coagulant therapy. Such complications are thought to be caused by high 
blood shear stresses, turbulence, as well as overall complexity of the haemodynamics in MHVs. Local high fluid stresses are recognised to be among the primary causes of haemolysis [2, 3] while organic material contained in blood can accumulate in flow stagnation or recirculation regions, leading to thrombus formation.

Numerical simulations can provide a deeper insight into the flow and shear stress patterns in regions where physical measurement is very difficult if not impossible [4-6]. However, the use of CFD to investigate MHV mechanics presents a considerable challenge because of large rotations encompassed by leaflets, and the fully coupled FSI features required to completely represent the physical phenomenon.

In particular, in the opening phase leaflets move under the ventricular pressure, raising above the aortic pressure at the beginning of systole. After reaching the maximum opening angle, depending on the valve mechanical constraints, leaflets remain in the fully open position (apart from observed small oscillations) until the ventricular pressure decreases below the aortic value after the systolic peak, causing the start of the closing phase. After complete closure leaflets remain in the fully closed position with some flow leakage occurring through the valve, until the aortic pressure becomes higher than the ventricular pressure up to the beginning of the following systole causing a new opening phase.

\section{Valve description}

A Sorin Bicarbon SIZE 27 bileaflet MHV has been considered in the study. The valve is composed of a titanium alloy housing, to which two pyrolytic carbon leaflets are hinged through a mechanism ensuring a rolling-without-sliding motion in order to minimize friction between the pivot and the housing recess. Leaflets have a curved profile, and separate the valve orifice into three areas offering equal resistance to the blood flow, in such a way to provide a uniform pressure profile and minimise turbulence on the outflow side. The valve housing is attached to the heart inner wall by means of a composite sewing ring, intended to ensure tissue in-growth, minimizing the possibility of perivalvular leakage.

The valve geometry and schematic flow pattern are shown in Figure 1. The outer diameter of the housing (B) is $27.8 \mathrm{~mm}$, while the inner diameter of the valve orifice varies along the housing height, having a smallest value (A) of 23.3 $\mathrm{mm}$. The overall orifice area is $4.14 \mathrm{~cm}^{2}$. The rotation angle $\theta$ of the leaflets with respect to the valve axis is $70^{\circ}$ in the fully closed position and $10^{\circ}$ in the maximum opening position. Therefore, the maximum leaflets rotation angle is $60^{\circ}$.

\section{Numerical simulation}

\subsection{Model description}

The computational domain of the numerical model coincides with the region of the experimental mock-loop including the leaflet housing, the aorta sinus, and 
two upstream and downstream cylindrical duct sections allowing the application of boundary conditions at sufficient distance from the area under investigation (see Figure 2-a). The overall domain length is $73.2 \mathrm{~mm}$. Due to the symmetry with respect to a plane bisecting one of the sinus lobes, only half of the model was considered for computation purposes.

Due to the large leaflet displacement during the valve opening and closing, a remesh algorithm needs to be adopted to progressively adapt the grid to the new position. In order to allow automatic remeshing, the grid was composed by tetrahedral elements in the zone interested by the leaflet motion and in the external region of the aorta sinus (with a dimension progressively increasing away from the leaflet surfaces), and hexahedral elements in the central part of the sinus and in the inlet and outlet cylindrical sections (see Figure 2-b).

Each leaflet (see Figure 3) has been considered to be composed of rectangular and semi-circular curved plates joined together, whose bending radius is approximately $28 \mathrm{~mm}$.
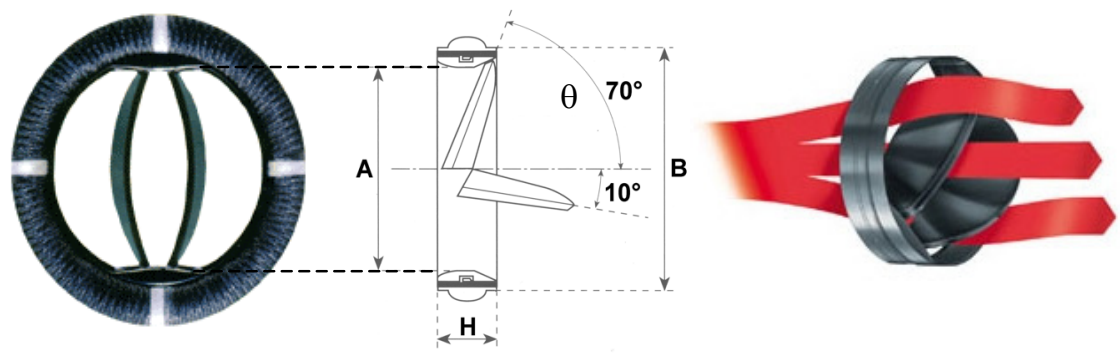

Figure 1: Valve geometry.

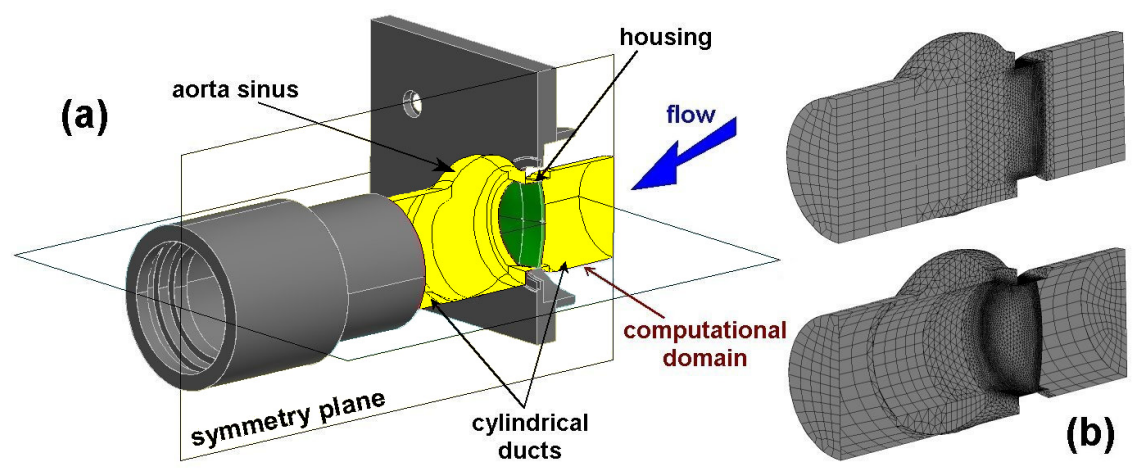

Figure 2: Computational domain. 


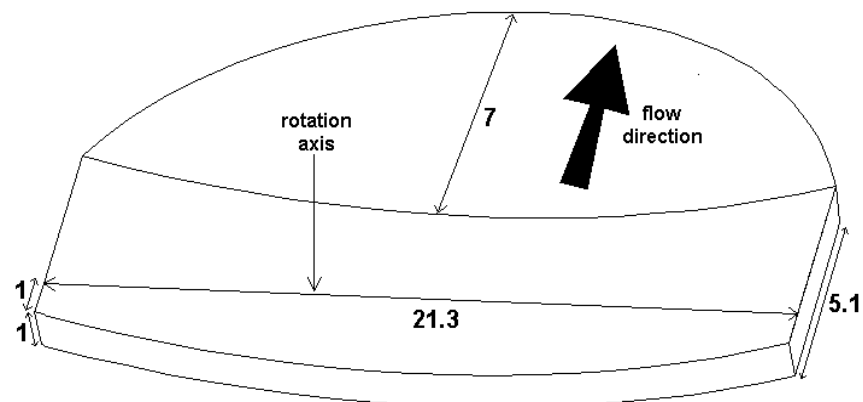

Figure 3: $\quad$ Leaflet dimensions ( $\mathrm{mm})$.
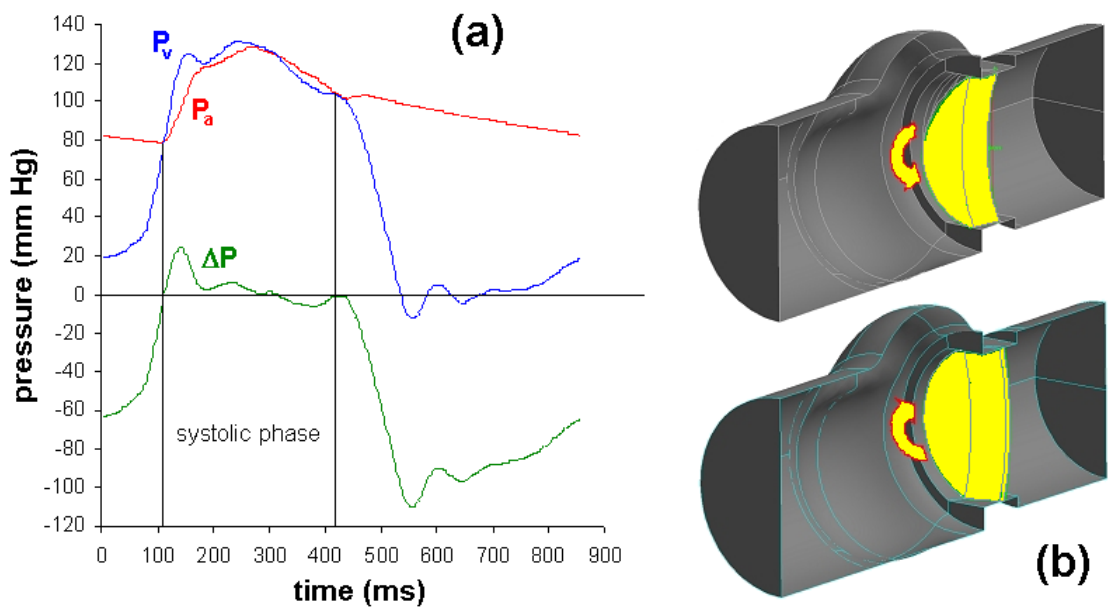

Figure 4: Simulation scenario.

\subsection{Simulation scenario}

The simulation starts at the beginning of systole, when the valve is fully closed and the left ventricle pressure $P_{v}$ rises above the aortic value $P_{a}$ (see Figure 4-a), and ends when the fully closed position is achieved again in correspondence of the ventricular pressure final drop below the aortic value at the end of systole (see Figure 4-b). A pressure profile corresponding to the difference $\Delta \mathrm{P}$ between the left ventricle and aorta values during the whole systolic phase is applied to the inlet section of the upstream duct.

Remeshing parameters were optimised in order to keep cells dimension as close as possible to the original value, minimising at the same time their skewness. In addition, a high grid resolution was kept only in those areas close to the leaflet surface where more accurate flow calculation is needed, whilst remeshing was avoided in the zones not interested by leaflet motion in order to save computational time. 
Flow was assumed to be incompressible with a density of $1056 \mathrm{~kg} / \mathrm{m}^{3}$ and a viscosity of $0.0035 \mathrm{~kg} / \mathrm{m} \cdot \mathrm{s}$, representative of human blood properties at $37^{\circ} \mathrm{C}$. In order to account for turbulent flow features the Standard $\mathrm{k}-\varepsilon$ turbulence model was applied, as being recognised to provide good results in turbulent simulation of blood flow in artificial heart devices [7].

\subsection{Fluid-Structure Interaction}

Direct calculation of the motion of a body within a fluid according to Newton's dynamics equation leads to unstable motion, which is further enhanced in the case of a solid body moving into a fluid duct if a small gap exists between the body surface and the duct walls [8]. In the case of a MHV motion such instability is particularly critical at the beginning of opening and at the end of the closing phase, when the leaflet tip gets very close to the housing ring.

In order to obtain a stable calculation, the leaflet position equilibrating the forces generated by the flow pressure has been derived at each timestep through the iterative FSI algorithm. To this purpose, a specific Valve Cycle Program (VCP) was developed and coupled to the commercial CFD solver (Fluent, Ansys Inc.).

The VCP logic is shown in Figure 5. In particular, the leaflet position ensuring the equilibrium between flow and inertial forces is recalculated until the absolute difference between the pressure moment on the leaflet surface and the inertial term of the motion equation is smaller than a tolerance value $\varepsilon$. Some of the operations to be performed in the FSI algorithm were implemented by means of dedicated User-defined Routines (USR).

Due to the variable leaflet velocity during the valve opening-closing cycle, the VCP automatically calculates the timestep size so that the overall angular displacement in each timestep is always compatible with the remeshing requirements. In addition, the leaflet opening angle $\theta$ is checked at each timestep, in order to stop FSI calculations when the maximum allowed value of $60^{\circ}$ is attained. From this point on steady iterations are performed with the leaflet position fixed at the maximum opening angle $\theta_{\max }$, until the pressure moment reverses causing restart of FSI iterations in the closure phase.

\section{Experimental flow measurements}

Flow measurements were performed by inserting the MHV in a realistic glass blown aortic root model specifically realised to mimic physiologic conditions. In turn, the aortic model was installed in a dedicated circulatory mock-loop employed as a hydraulic model of the left human circulatory system, able to generate physiological pressure and flow waveforms in the aortic valve position.

A 35\% water-glycerine solution, featuring blood analogue density of 1,09 $\mathrm{g} / \mathrm{cm}^{3}$ and dynamic viscosity of $3,57 \mathrm{mPa} \cdot \mathrm{s}$, was chosen as testing fluid. The fluid selection was also driven by the necessity of having a refraction index matching as close as possible to that of the surrounding material. 


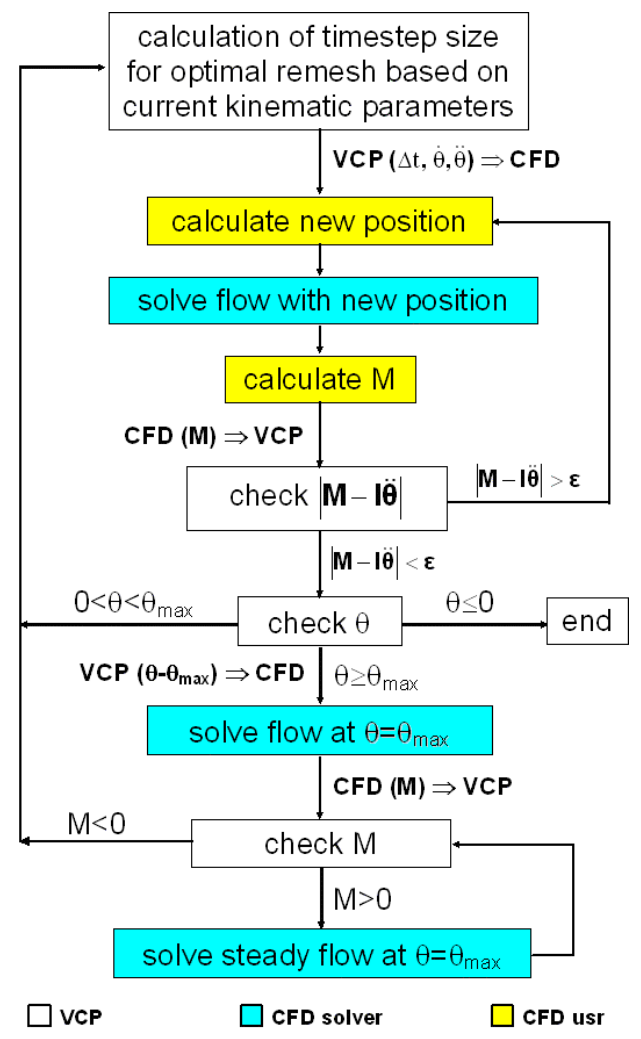

Figure 5: $\quad$ FSI implementation.

Flow data were acquired by means of a 2D PIV system, composed by a 532 $\mathrm{mm}$ wavelength double cavity pulsed Nd-Yag laser generating a light sheet with a thickness of about $150 \mu \mathrm{m}$, and a 1376x1040 CCD camera capturing the laser light scattered by $10 \mu \mathrm{m}$ fluorescence seeding particles suspended in the fluid (see Figure 6).

The laser sheet was aligned with the cross section of the aortic root model to be investigated, while the camera was arranged at a $90^{\circ}$ angle with respect to the light sheet (see Figure 7). The operating point of the circulatory mock-loop was set to a cycle rate of 70 beats per minute, a cardiac output of $5 \mathrm{l} / \mathrm{min}$ and a systolic duration of $35 \% \pm 2 \%$, in accordance to admission procedures defined by US Food and Drug Administration (Heart Valve Guidance) and European standardisation authority (DIN EN ISO 5840).

The flow field downstream the valve was investigated in six different planes at $3 \mathrm{~mm}$ incremental distance from the aortic root center cross section $(\mathrm{z}=0,3$, $6,9,12,15$ and $18 \mathrm{~mm}$ in Figure 7). In each plane flow data were acquired at 16 different time instants after the start of the opening phase, corresponding to specific leaflet opening angles. In order to ensure a significant statistics 
averaging, 300 acquisitions were performed at each of the 16 angular positions by opportunely triggering the system for periodic images recording at the desired phase angle in successive opening-closing cycles.



Figure 6: Experimental equipment.
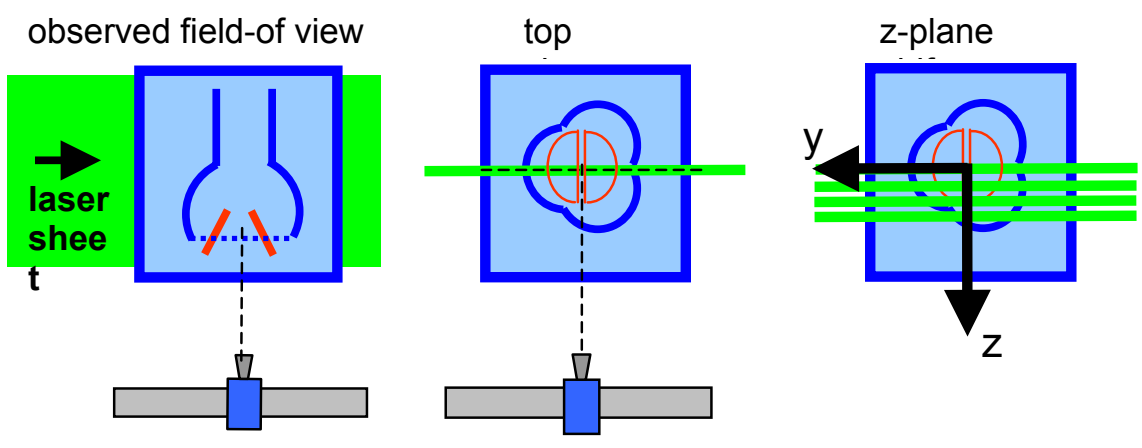

Figure 7: $\quad$ PIV system setup. 


\section{Comparison of numerical and experimental results}

The calculated time history of leaflet angular velocity and opening angle is shown in Figure 8. The valve reaches the maximum opening angle after about 40 $\mathrm{ms}$ from opening start, remaining in this position for approximately $155 \mathrm{~ms}$ with small oscillations around $100 \mathrm{~ms}$, to start then the closing phase lasting about 95 ms. The maximum angular velocity is around $50 \mathrm{rad} / \mathrm{s}$ both in opening and closing phases. The predicted cycle dynamics result to be in good agreement with experimental fast camera recording, including oscillations during the permanence at maximum opening angle, as well as with data reported in the literature for similar valves (for instance St. Jude type [9]).

The flow field downstream the valve can be spatially characterised into different areas with peculiar features (see Figure 9). The open leaflets clearly separate the flow in three main jets over an axial extension of about $20 \mathrm{~mm}$ immediately downstream the valve (zone 1), progressively diffusing into each other at moving away (zone 2). The lateral confinement exerted by the side jets (zones B) on the larger central jet (zone C) leads it to extinguish at a distance of approximately $50 \mathrm{~mm}$ from the valve (zone $\mathrm{C} 2$ ). In the radial peripheral region (zones A) the flow velocity gradually approaches a zero value consistently with the wall no-slip condition. Reverse flow due to recirculation induced in the aorta sinus (see Figure 7) is clearly visible in zone A1.
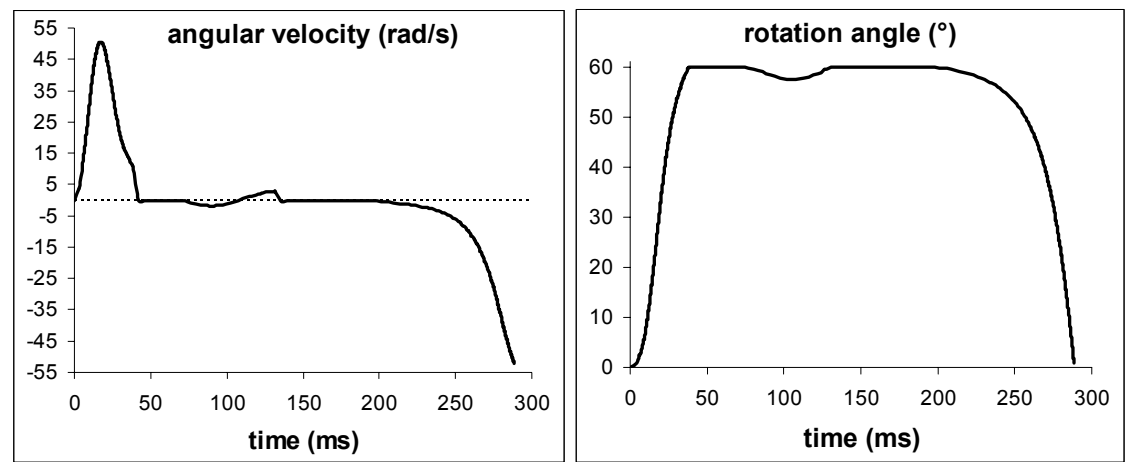

Figure 8: Predicted valve kinematics.

The percentage difference between experimental and numerical average flow velocity in each of the identified zones is reported in Table 1 . A very close match was generally observed in zones $\mathrm{B}$ and $\mathrm{C}$, with a slightly lower agreement in zone $\mathrm{C} 2$ probably due to the effect of the asymmetric experimental configuration (see Figure 7) with respect to the symmetric numerical model (see Figure 4). The higher differences in zones A, keeping anyway within acceptable limits, are most likely caused by the insufficient PIV resolution to accurately capture the large flow gradients occurring in the proximity of the walls. 


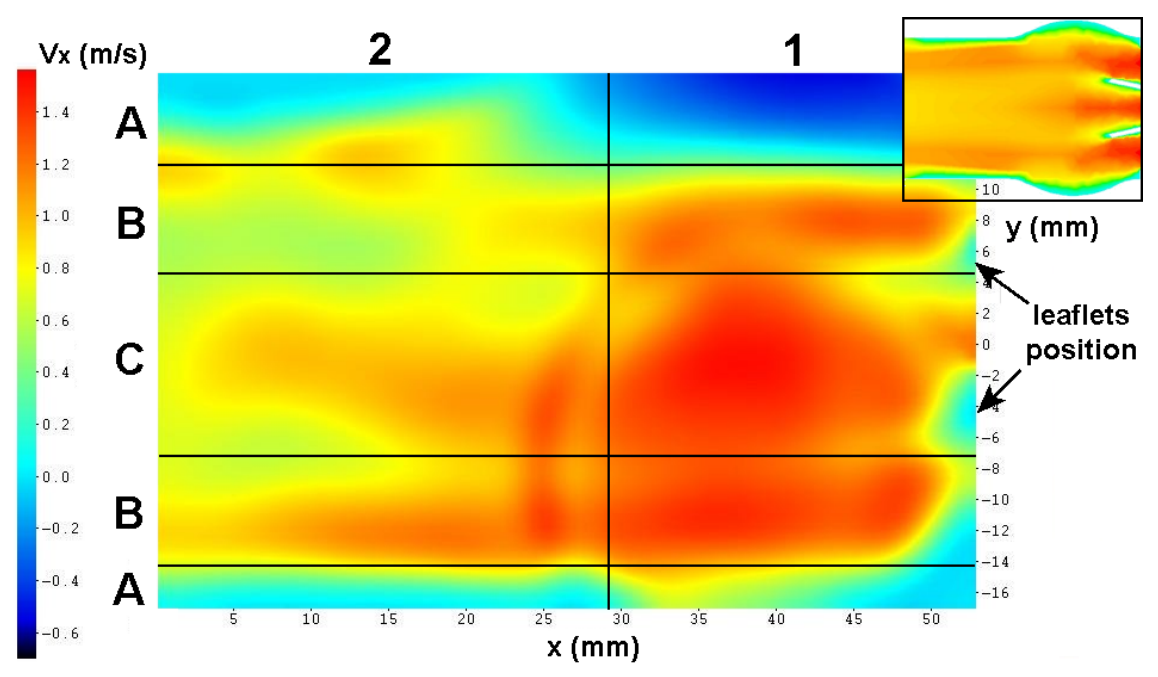

Figure 9: Experimental axial velocity field at $\mathrm{z}=3 \mathrm{~mm}, \mathrm{t}=110 \mathrm{msec}$ from opening start (see in top-right box an example of numerical output).

Table 1: Result of comparison between numerical and experimental outputs.

\begin{tabular}{|c|c|c|c|}
\hline Zone & $\mathrm{A}$ & $\mathrm{B}$ & $\mathrm{C}$ \\
\hline 1 & $8 \%$ & $2 \%$ & $2 \%$ \\
\hline 2 & $10 \%$ & $3 \%$ & $5 \%$ \\
\hline
\end{tabular}

\section{Conclusions}

A numerical methodology to enable full FSI simulation of complete opening and closing of a MHV was developed and coupled to the commercial CFD code Fluent (Ansys Inc.) as a completely automated procedure. The predicted results were found to agree with the corresponding PIV flow measurements performed on a specifically realised circulatory mock-loop.

Both numerical and experimental results showed a thoroughly rinse of the area downstream the valve, indicating a low thrombosis potential. In addition, flow shear stresses lower $200 \mathrm{~Pa}$ were observed in all investigated section planes at any time of the opening-closing cycle, suggesting that also haemolysis potential is not critical.

However, additional analysis will be developed to confirm low haemolysis and thrombosis potential in flow areas around the valve, as demonstrated by clinical tests (SORIN). Regions around the leaflet hinge to the housing have been highly jeopardised in past concept valves to induce thrombus formations if a thoroughly rinse was not ensured. Other critical areas with respect to shear stress values were the tips of the leaflets, where small vortices may cause high erythrocyte exposure especially during the final closure when a very small gap exists with the valve housing. In order to enable an accurate flow 
characterisation in such small regions the grid resolution has to be consistently increased, which in turn requires a corresponding enhancement of computational resources.

\section{Acknowledgement}

Results of present work have been achieved in the frame of the European Commission FP6 funded project, Smart-PIV (IST-2001-37548 contract).

\section{References}

[1] Yoganathan, A. \& Sotiropoulos, F., Using CFD to Examine the Haemodynamics of Artificial Heart Valves. Business Briefing: US Cardiology 2004.

[2] Garon, A. \& Farinas, M., Fast Three-dimensional Numerical Haemolysis Approximation. Artificial Organs, 28(11), pp. 1016-1025, 2004.

[3] Lu, P.C., Lai, H.C. \& Liu, J.S., A Reevaluation and Discussion on the Threshold Limit for Haemolysis in a Turbulent Shear Flow. J. Biomech., 34(10), pp. 1361-1364, 2001.

[4] De Wachter, D. \& Verdonck, P., Numerical Calculation of Haemolysis Levels in Peripheral Haemodialysis Cannulas. Artificial Organs, 26(7):576-582.

[5] Apel, J. et al, Assessment of Hemolysis Related Quantities in a Microaxial Blood Pump by Computational Fluid Dynamics. Artificial Organs, 25(5):341-347.

[6] Song, X. et al, Quantitative Evaluation of Blood Damage in a Centrifugal VAD by Computational Fluid Dynamics. Journal of Fluids Engineering, May 2004, Vol. 126:410-418.

[7] Palmieri D. et al, A Methodological Approach for the Optimisation of Modern Rotary Blood Pumps. Proceedings of VI International Conference on Modelling in Medicine and Biology, Bologna, Italy, 7-9 September 2005, pp. 231-240.

[8] Vierendeels, J. et al, Stabilization of a Fluid-Structure Coupling Procedure for Rigid Body Motion. 33 $3^{\text {rd }}$ AIAA Fluid Dynamics Conference and Exhibit, 23-26 June 2003, Orlando, Florida, AIAA 2003-3720.

[9] Penrose, M.T. \& Staple, C.J., Implicit Fluid-Structure Coupling for Simulation of Cardiovascular Problems. Int. J. Numer. Meth. Fluids, 40:467-478, 2002. 